EPJ Web of Conferences 19, 05006 (2012)

DOI: $10.1051 /$ epjconf/20121905006

(C) Owned by the authors, published by EDP Sciences, 2012

\title{
Chemical composition of stars in kinematical substructures of the galactic disk
}

\author{
T.V. Mishenina ${ }^{1, a}$, C. Soubiran ${ }^{2}$, S.A. Korotin ${ }^{1}$, T.I. Gorbaneva ${ }^{1}$ \\ and N. Yu Basak ${ }^{1}$ \\ ${ }^{1}$ Astronomical Observatory of Odessa National University, Odessa, Ukraine \\ 2 Universite de Bordeaux 1-CNRS-Laboratoire d'Astrophysique de Bordeaux, UMR 5804, \\ France
}

\begin{abstract}
The $\mathrm{Y}, \mathrm{Zr}, \mathrm{La}, \mathrm{Ce}, \mathrm{Nd}, \mathrm{Sm}$ and $\mathrm{Eu}$ abundances were found in LTE approach, and the abundance of Ba was computed in NLTE approximation for 280 FGK dwarfs in the region of metallicity of $-1<[\mathrm{Fe}]<+0.3$. The selection of stars belonging to thin and thick disks and the stream Hercules was made on kinematic criteria. The analysis of enrichment of the different substructures of the Galaxy with $\alpha$-element $(\mathrm{Mg}, \mathrm{Si})$, the iron peak $(\mathrm{Ni})$ and neutron-capture elements was carried out.
\end{abstract}

\section{INTRODUCTION}

The kinematical and chemical distributions of the old stars of the galactic disk show evidence of at least 3 substructures : the thin disk, the thick disk and the Hercules stream [6]. One way to investigate the processes which formed the different structures of the disk is to study in details the chemical composition of the stars belonging to these structures. $\alpha$-, n-capture, iron-peak elements are formed under the different conditions in objects of different masses and at various stellar lifetimes. The yields of these elements and their abundances can thus be used to investigate how the Galactic substructures and the Galaxy in general have evolved and were enriched by these elements.

\section{OBSERVATIONS, SELECTION AND PARAMETERS OF PROGRAM STARS}

Observations of 280 stars have been carried out with the $1.93 \mathrm{~m}$ telescope at OHP (France) equiped with the ELODIE echelle-spectrograph. The spectra cover the wavelength range $440-680 \mathrm{~nm}$ at a resolution of 42000 , with typical $\mathrm{S} / \mathrm{N}$ at $550 \mathrm{~nm}$ of 100 to 350 . The probability of each star to belong to the thin, thick disk and the Hercules stream was computed using the (U, V, W) velocities following the method of [6]. The parameters $\left(T_{\text {eff }}, \log g, V_{t},[\mathrm{Fe} / \mathrm{H}]\right)$ and $\mathrm{Mg}, \mathrm{Si}, \mathrm{Ni}$ abundances were estimated earlier in our papers [3, 4]. Y, Zr, La, Ce, Nd, Sm abundances were determined for 280 of FGK dwarfs in LTE approximation, with the WIDH9 code and the atmosphere models by Kurucz [2]. Determination of the Eu abundance was made by the STARSP LTE spectral synthesis code [7] taking into account the hyperfine structure. The abundance of barium was computed in NLTE approach using the Ba II 4554, 5853,6141 and $6496 \AA$ lines.

\footnotetext{
a e-mail: tmishenina@ukr.net
}

This is an Open Access article distributed under the terms of the Creative Commons Attribution-Noncommercial License 3.0, which permits unrestricted use, distribution, and reproduction in any noncommercial medium, provided the original work is properly cited. 

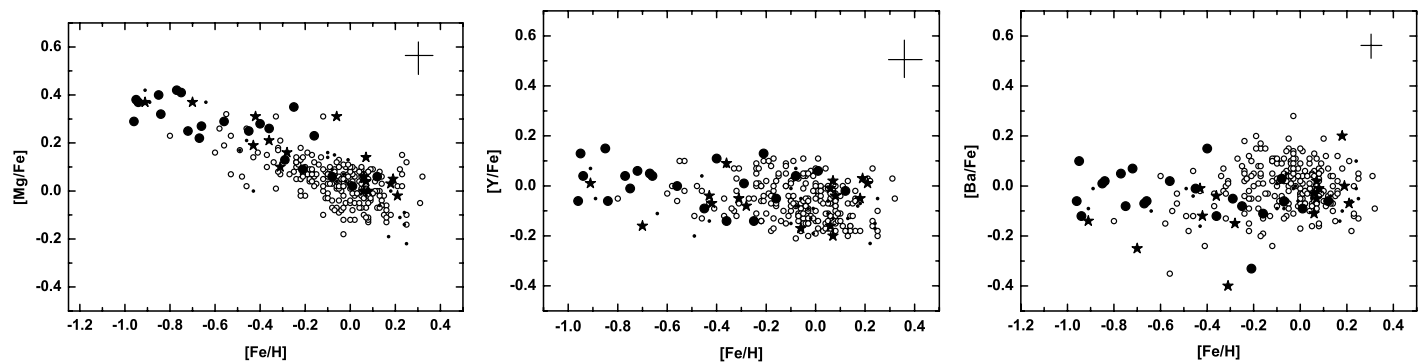

Figure 1. The dependences of $[\mathrm{Mg} / \mathrm{Fe}]$, $[\mathrm{Y} / \mathrm{Fe}],[\mathrm{Ba} / \mathrm{Fe}]$ upon $[\mathrm{Fe} / \mathrm{H}]$.
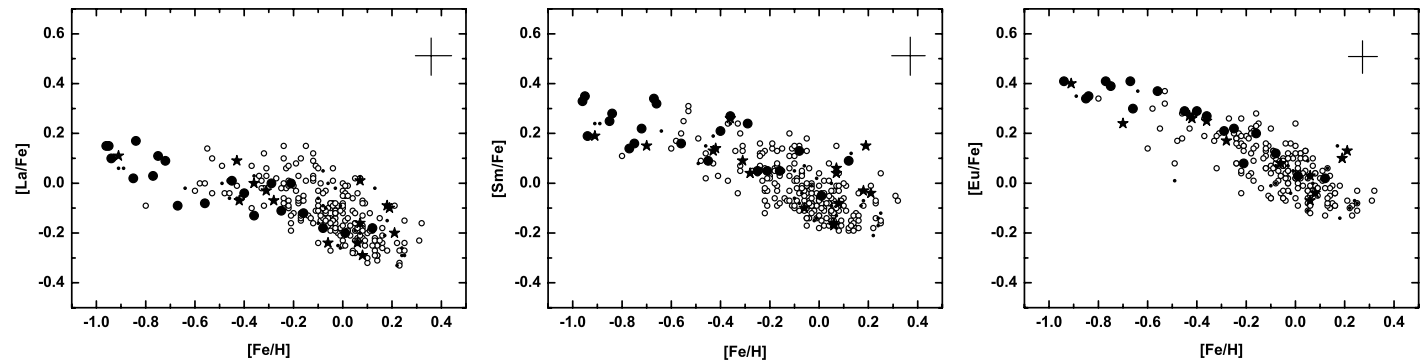

Figure 2. The dependences of $[\mathrm{La} / \mathrm{Fe}],[\mathrm{Sm} / \mathrm{Fe}]$, $[\mathrm{Eu} / \mathrm{Fe}]$ upon $[\mathrm{Fe} / \mathrm{H}]$.

\section{RESULTS AND CONCLUSIONS}

The abundance trends $[\mathrm{El} / \mathrm{Fe}]$ vs. $[\mathrm{Fe} / \mathrm{H}]$ are presented in Figs 1,2 . The stars are marked by open (thin disk) and filled (thick disk) circles, asterisks (Hercules stream) and small points (unclassified). Our sample shows that there are thick disk stars at solar metallicity and that the thin disk has a metal-poor tail down to $[\mathrm{Fe} / \mathrm{H}] \backsim-0.80$. At solar metallicity, thin and thick disk stars have the same chemical behaviour. For some elements there is a clear change of trend at $[\mathrm{Fe} / \mathrm{H}] \backsim-0.30$. This is specially visible for $\mathrm{Ba}$, $\mathrm{La}$ and $\mathrm{Nd}$. Clearly $\mathrm{Mg}$ is decreasing with increasing metallicity, confirming previous results on $\alpha$-elements $([1,3,5,6]$, etc). Thick disk stars show higher values of $[\mathrm{Mg} / \mathrm{Fe}]$ than thin disk stars, even in the metallicity range where both disk overlap greatly $(-0.50<\mathrm{Fe} / \mathrm{H}]<0)$. $[\mathrm{Mg} / \mathrm{Fe}]$ for the stars of Hercules stream spans all the values of both disks. $[\mathrm{Si} / \mathrm{Fe}]$ vs. $[\mathrm{Fe} / \mathrm{H}]$ shows a similar behaviour as $[\mathrm{Mg} / \mathrm{Fe}]$ but with a significant lower dispersion and smaller slope. Nickel, an iron-peak element, provides a distribution centered on $[\mathrm{Ni} / \mathrm{Fe}]=0$, slightly rising at $[\mathrm{Fe} / \mathrm{H}]>0$, and with a remarkable low dispersion $\mathrm{Y}$ and $\mathrm{Zr}$ demonstrate different trends with $[\mathrm{Fe} / \mathrm{H}]$ : no dependence of $[\mathrm{Y} / \mathrm{Fe}]$ with $[\mathrm{Fe} / \mathrm{H}]$ is observed, but we see an increase of $\mathrm{Zr}$ abundance with decreasing $[\mathrm{Fe} / \mathrm{H}]$ below -0.3 . $[\mathrm{Ba} / \mathrm{Fe}]$ vs. $[\mathrm{Fe} / \mathrm{H}]-$ The trend obtained for the Ba abundance versus $[\mathrm{Fe} / \mathrm{H}]$ suggests a complex barium production process in the thin and thick disks. $[\mathrm{La} / \mathrm{Fe}]$ shows a slight trend with $[\mathrm{Fe} / \mathrm{H}]$ with remarkable deficit of $\mathrm{La}$ abundance at $[\mathrm{Fe} / \mathrm{H}]>0$. Ce abundance behaves similarly in all substructures, there is no trend with $[\mathrm{Fe} / \mathrm{H}] .[\mathrm{Nd} / \mathrm{Fe}]$ vs. $[\mathrm{Fe} / \mathrm{H}]$ for the thick disk stars does not show any trend with $[\mathrm{Fe} / \mathrm{H}]$, but at $[\mathrm{Fe} / \mathrm{H}]>-0.3$, the $\mathrm{Nd}$ abundance increases with decreasing $[\mathrm{Fe} / \mathrm{H}] .[\mathrm{Sm} / \mathrm{Fe}]$ vs $[\mathrm{Fe} / \mathrm{H}]$ shows increasing values of $\mathrm{Sm}$ abundances with decreasing metallicity for all substructures and higher values for thick disk stars. Europium, an element formed predominantly in $\mathrm{r}$ process, shows a marked trend with $[\mathrm{Fe} / \mathrm{H}]$ and a slight increase of abundance for thick disk stars. 
Assembling the Puzzle of the Milky Way

\section{References}

[1] Bensby et al., A\&A 433, (2005) 185

[2] Kurucz R.L., CD ROM n13 (1993)

[3] Mishenina T.V., Soubiran C., Kovtyukh V. V., Korotin S.A., A\&A 418, (2004) 551

[4] Mishenina, T.V., Soubiran C., Kovtyukh V. V., et al., A\&A 489, (2008) 923

[5] Reddy et al., MNRAS 367, (2006) 1329

[6] Soubiran, C., Girard, F., A\&A 438, (2005) 139

[7] Tsymbal, V.V., ASP Conf. Ser. 108, (1996) 198 